\title{
Parent perspectives on children and young people's mental health services in Victoria - what's wrong and how to fix it: A multi-site qualitative study.
}

\section{ABSTRACT \\ Objective}

The number of children and young people presenting to emergency departments (ED) with anxiety and depression is increasing. We aimed to determine parent perspectives on (i) barriers to accessing non-ED mental health services and (ii) improving access in the paediatric mental health service system.

\section{Methods}

Qualitative study with parents of children and young people aged 0-19 years who attended one of four emergency departments across Victoria between October 2017 and September 2018 and received a primary diagnosis of anxiety or depression. Exclusion criteria: child or young person without a parent/guardian, or presented with self-harm or suicide attempt. Eligible participants completed semi-structured phone interviews. Interviews were recorded and transcripts were coded and analysed using content analysis.

\section{Results}

72 parents completed interviews. The average child age was 14 years (standard deviation 2.5) and two thirds identified as female (64\%). 57\% of children and young people presented with a primary diagnosis of anxiety. Parents reported barriers in accessing care including: service shortages and inaccessibility; underresourced schools; lack of clinician mental health expertise; lack of child-clinician rapport; inconsistent care; financial constraints; lack of mental health awareness among parents; and stigma. Parents want expanded and improved access to services; more respite and support services; supportive schools; and improved mental health education for parents.

\section{Conclusions}

This is the author manuscript accepted for publication and has undergone full peer review but has not been through the copyediting, typesetting, pagination and proofreading process, which may lead to differences between this version and the Version of Record. Please cite this article as doi: 10.1111/jpc.14835

This article is protected by copyright. All rights reserved. 
Parents of children and young people attending the emergency department for anxiety and depression are generally dissatisfied with services for child mental health. Solutions that enable parents to better care for their child in the community are needed to improve care.

\section{Key words}

Anxiety; Child; Adolescent; Depression; Health Services Accessibility; Mental Health Services

\section{WHAT IS ALREADY KNOWN}

1. The rate of paediatric anxiety and depression presentations to health professionals and Emergency Departments (EDs) is rising.

2. This is despite the fact that the availability of psychological and pharmacological treatments has increased.

3. Understandings of parental perspectives' on barriers to community mental health care and what an ideal mental health system would look like, can inform policy to improve access to care.

\section{WHAT THIS PAPER ADDS}

1. In a multi-site study of parents of children and young people attending ED for anxiety or depression, barriers to accessing care included: service shortages and inaccessibility; underresourced schools; lack of clinician mental health expertise; lack of child-clinician rapport; inconsistent care; financial constraints; lack of mental health awareness among parents; and stigma.

2. Parents say that an ideal system would include expanded and improved access to services; more respite and support services; supportive schools; and improved mental health education for parents.

This article is protected by copyright. All rights reserved. 


\section{INTRODUCTION}

Australia has significantly increased its investment in mental health care over recent decades, ${ }^{1,2}$ with a deliberate focus on improving adolescent mental health services. ${ }^{3}$ However, despite these investments, the number of children and young people aged 10-18 years presenting to Victorian emergency departments (ED) with mental health problems has increased particularly those who received a diagnosis of anxiety or depression. ${ }^{4}$

A systematic review of 44 studies examined parents' perceptions of barriers and facilitators to accessing treatment for mental health problems in children and adolescents. Recurrent barriers included long waiting times, lack of specialist services, restrictive referral criteria, feeling dismissed by professionals, parent difficulties identifying a mental health problem and not knowing where or how to seek help. ${ }^{5}$ This review included two Australian studies - one a cross sectional survey ${ }^{6}$ and the other a small qualitative study of 15 parents of children attending mental health services other than the ED. ${ }^{7}$

It is important to understand whether the parent-perceived barriers outlined above are relevant to the increase in child and adolescent mental health presentations to the ED for anxiety and depression. Further, it is important to understand what parents, as key consumers of the mental health system, believe an ideal mental health service might entail for their child. We therefore aimed to explore parent perceived barriers to accessing non-ED services, and parent perspectives on the attributes of an ideal service system.

\section{METHODS}

This was a multi-site, qualitative study in four Victorian public hospital ED settings (two major referral paediatric hospitals, one urban district mixed adult/paediatric and one regional mixed adult/paediatric ED). Approval to conduct the study was granted by the Royal Children's Hospital Human Research Ethics Committee (HREC 37147A). Each site provided site specific approval.

This article is protected by copyright. All rights reserved. 
We recruited a purposive sample of parents of children and young people aged 0-19 years who presented to ED from October 2017 to September 2018. Inclusion criteria wereparents of children and young people who received an ED discharge diagnosis from the treating ED doctor of 'Mood (Affective) Disorder' or 'Neurotic, Stress-related or Somatoform Disorder', as classified by the International Classification of Diseases 10th edition, Australian Modification (ICD-10-AM), together with a clinician diagnosis of anxiety or depression. Exclusion criteria are detailed in Table 1.

ED clinicians at each site identified 135 potentially eligible families through a rolling series of electronic medical record searches for the discharge diagnosis. All eligible families were mailed a recruitment letter by the participating hospitals. Families were given two weeks to opt out of hearing more about the study. If they did not, the ED clinician passed the family contact details to the research team.

Three researchers (ASC, KD and RL), with a Master of Public Health, a Master of Social Work, and a FRACP trainee respectively, undertook training in study protocols, interviewing techniques specific to this study and pilot interviews prior to commencing interviews with participants. Researchers then telephoned parents to explain the study, confirm eligibility, obtain verbal consent and conduct the interview.

All participants were asked a series of questions from a semi-structured interview script (Table 2) however, researchers were also allowed sufficient flexibility for exploration of emerging themes. ${ }^{8}$ Field notes were kept for all interviews. With permission from the participants, interviews were audio recorded, transcribed, reviewed for accuracy by two of the authors (ASC, KD), deidentified and imported into NVivo version 12 Analysis Software (QSR International Pty Ltd. Cardigan UK) for analysis.

An audit trail of study decisions was kept by the research team. Reflexivity was maintained through weekly discussions with the research team and journaling by individual researchers.

\section{Data analysis}


Child and family descriptive measures (e.g. age, sex, presentation details) are presented as the number and proportion of presentations. Child home postcode was mapped to the Australian Bureau of Statistics (ABS) 2011 Remoteness Areas. ${ }^{9}$ We adopted a descriptive phenomenological approach as our methodological framework, to understand the experiences of parents or carers of children and young people living with anxiety and depression. ${ }^{10,11}$ The broad aims of the research question provided the broad focus for analysis but more "interpretive" sub-themes were developed using an inductive approach. Categories were developed in line with the primary research questions and themes were then identified using the processes of content analysis. ${ }^{12}$

Three researchers (ASC, KD and RL), conducted the initial analysis, compared codes for consistency and discussed discrepancies with HH to develop a coding schema which was then applied to all transcripts.. The research team met regularly to discuss emerging categories and patterns in the data and to reach consensus on major themes. ${ }^{13}$ We interviewed all those who consented to take part and this allowed for a rich description on caregiver perspectives on the research questions.

\section{RESULTS}

A total of 72 interviews (of 92 eligible and contacted) were completed, ranging in length from 25-60 minutes. 15 families (21\%) were living in a regional area. Most (85\%) study participants were female. Child and young person ages ranged between 8 and 18 years and two thirds were female (64\%). 57\% of children and young people had a primary ED discharge diagnosis of 'Neurotic, Stress-related or Somatoform Disorder' and 43\% a diagnosis of 'Mood (Affective) Disorder'. Approximately half of all participants had been referred to the ED (53\%), or presented during standard business hours (47\%), and more than a third (39\%) had previously presented to the ED with mental health concerns.

Analysis of the interview transcripts revealed some important themes which are presented using deidentified verbatim representative quotes. Themes could be broadly categorised into : 
1. Understanding why parents bring their child or young person to ED for mental health issues, and

2. What are the barriers to receiving services in the community and how to improve access to services

This paper reports on a subset of the themes from Theme 2. Barriers to accessing services and how to improve mental health services for children and young people.

Parents' perceptions of the barriers to accessing treatment for mental health issues in children andyoung people, and the attributes of an ideal service environment, could be summarised under three key overarching themes: (1) systemic factors within healthcare and schools; (2) clinician practice attributes and relationship with child/family; and (3) family/child factors. Ideal service environments' overarching themes were: (1) Systemic factors within healthcare \& schools and (2) family and child factors

\section{Barriers to accessing treatment}

\section{Systemic factors within healthcare \& schools}

\section{Service shortages}

Parents reported limited service availability as the most significant barrier to securing care and they were frustrated to discover many mental health professionals had either closed their books to new patients or had long waiting lists for an initial appointment.

When we were trying to find a psychiatrist, we were in desperation and we had to wait eight weeks. That was for a private psych. ... which surprised me because $<$ now $>$ she's already a patient, I could get an appointment next week. An appointment is an appointment whether it's a new patient or an existing patient. ... (daughter, 15 yrs)

They found long delays in securing community-based child and adolescent mental health service (CAMHS) appointments particularly frustrating and many of these families resorted to 
seeing a clinician privately. For those families who had already seen a mental health professional, parents complained of protracted wait times between appointments and limited service availability after hours and during school holidays.

Parents of children and young people with chronic mental health issues reported a noticeable lack of child and adolescent psychiatric inpatient units. Thus, parents of adolescents in acute psychiatric distress often present to ED in desperation, hoping their child might be fast-tracked to an inpatient unit. Parents of young children (i.e., under 12 years) requiring acute intervention felt particularly isolated and reported presenting to the ED as their last resort.

In the absence of actually articulating any kind of suicidal thoughts, the practitioner told her that because she wasn't in that extreme situation that there was no chance she'd be admitted to the hospital. Which is what she was desperate for. She wanted respite from herself ... and she got told very quickly and very definitely that she wouldn't be getting anything. (daughter, 16 yrs)

Families living in regional areas also encountered many challenges accessing care. Parents complained the services were insufficiently resourced and yet commuting for metropolitan services was out of reach for some, given the lack of services available after hours. However, one of the greatest challenges reported by parents living regionally was the absence of child and adolescent inpatient psychiatric facilities. Some families whose children needed inpatient servies were admitted to the nearest adult inpatient psychiatric unit which parents believed was not only inappropriate and confronting for adolescents but often gave rise to other issues (e.g., undesirable relationships developing between adolescents and adult inpatients) which families then had to navigate.

They don't have an adolescent mental health unit ... they're really just a crisis centre. There's no therapy there and it's not a very nice place for teenagers so ... if something happens with CHILD, even though Monash is an hour and a half away, I take him <there > because they have a psych there 24 hours and because they have paediatric and because it's the door into $<$ inpatient psychiatric facility> if he needs it. (son, 17 yrs) 
Parents of children and young people with autism-spectrum disorder (ASD), attention-deficithyperactivity disorder (ADHD) and/or obsessive-compulsive disorder (OCD) reported the highest levels of distress in terms of accessing appropriate care. They reported significant difficulties finding mental health services and inpatient facilities that could cater to both their child's cormobidity and mental health issue. They often described feeling dismissed or unsupported by community health professionals.

He wouldn't engage < with> almost everyone I took him to in terms of a mental health person. He wasn't really physically unwell. A paediatrician didn't really have a role. There definitely should have been someone looking after him in terms of his mental health and his autism spectrum disorder but ... there's just no one around that does that. Instead, I just paid a lot of money to put him in a private school that could give him extra support and kind of manage the bullies a bit better than<the> state system could. (son, 16 yrs)

Service accessibility including costs, operating hours, and eligibility criteria

Many parents were frequently exhausted by the effort and resources it took to find a suitable clinician or service and they were frustrated by resulting treatment delays. For many parents, the operating hours, location and expense of services services were a hindrance to ongoing and timely treatment of their child's condition.

I'm a working parent and trying to get CHILD to appointments around my work and her school and those things can just be a nightmare. (daughter, 17yrs)

Some families reported perceived difficulties gaining access to mental health services due to stringent and non-transparent eligibility criteria. For example, some parents were referred to community mental health services only to be informed their child's condition was not debilitating enough to warrant an appointment.

And a mental < health care> plan is not enough visits ... six and then another four. Then what happens after that? (son, 17 yrs) 
There was one Sunday night that he had run away and we couldn't find him for two hours. ... When we did find him he came back and said that he was going to try and drown himself in the creek. And then on another occasion, I caught him trying to take a knife into the bathroom. ... < The hospital > referred us to CAMHS ... They did an assessment with me over the phone about CHILD. They never actually came down to see him. They decided that he wasn't too much of a risk. (son, 17 yrs)

Some parents of adolescents aged 16 and over who had contacted their local crisis assessment team during an acute psychiatric crisis were refused service without adequate explanation.

I tried the CAT team, but they did nothing ... <on> several occasions. They said they wouldn't do anything because CHILD wouldn't talk to them but they could hear him yelling in the background. So they knew there was an issue but they did absolutely nothing. Their only solution was call the ambulance. Each time I called them, that's all they said. There was literally no help. (son, 17 yrs)

Parents of older adolescents perceived that, discharge from community mental health services at 18 years proved a disconcerting experience for some young people, particularly those who had comorbidities and who had established a healthy rapport with the treating health professional.

\section{Underresourced schools}

Some parents were critical of school services for failing to offer counselling services, lacking the mental health expertise to advise parents on suitable services, and not liaising with the parents of children who had disengaged from school.

I felt very disappointed with the school. They didn't follow up properly, and so CHILD lost connection with school and last year she missed all of year 11.... it's been terrible for CHILD. (daughter, 17 yrs)

\section{Clinician practice attributes and relationship with child/family}

Clinician mental health expertise

This article is protected by copyright. All rights reserved. 
Some parents described being frustrated by a perceived lack of mental health awareness and expertise among certain primary health care professionals, including failure to diagnose their child's physical symptoms as psychogenic, inadequate knowledge of mental health services available, and underestimating the severity of their child's mental health state.

One of the frustrating things was that the GP wasn't aware of children's mental health professionals that could help. These were people I found. I had to do the digging around. (son, 15 yrs)

\section{Inconsistent care}

It was usual practice for families to experience a variety of services and clinicians before finding a service and/or clinician with whom their child had a positive rapport. Some of these families felt compelled to pursue private treatment options as this was the only way they could guarantee a consistent clinician for their child and increase their child's prospects of staying engaged in treatment.

The problem I find is that you have to shop around to find somebody ... who CHILD will engage with. And you don't know until they've met them ... you could blow five weeks waiting to get into someone only to find that CHILD doesn't want to see them again. (daughter, 17 yrs)

I myself have been to many different counsellors and it becomes frustrating reliving traumatic events over and over again with counsellors that are sort of ineffective. I don't want her to have that experience, so just trying to find somebody with a good reputation as well definitely plays into that. (daughter, 14 yrs)

For other families, the lack of continuity in their child's care was an issue resulting from health professionals taking holidays and maternity leave, often precipitating an ED presentation.

Parental agency

This article is protected by copyright. All rights reserved. 
Some parents reported a perceived lack of agency in the relationship between their child and his/her clinician. While they understood the imperative of trust in this relationship, they equally believed the clinician's strict adherence to confidentiality denied them an opportunity to better understand both their child's experiences and how best to help their child between visits.

When she first started, she was only 13 years old, so I approached them with, "I want this to be a joint effort and I need a bit of feedback ... I didn't want to know what went on in the sessions but I just needed to know if she was okay and what I needed to be doing. ... I didn't get any feedback at all, no matter how hard I tried. (daughter, 14 years)

\section{Family/child factors}

\section{Financial constraints}

Many parents complained of the financial burden associated with accessing ongoing treatment and the inadequacy of the Medicare contribution to cover out-of-pocket costs.

Our finances were pretty tight because I've also got two boys who are ASD/ADHD. ... And of course they all need therapies and it's 160 dollars an hour. ... And I have to choose between three of my children which one needs it the most. (daughter, 12 yrs)

Beyond consultation costs, parents identified other expenses such as being forced to reduce their employment commitments or pursuing private education for their child to secure schoolbased mental health services.

\section{Mental health literacy}

Some parents revealed they had been unaware their child was experiencing mental health issues, particularly parents of children with persistent physical complaints. Many parents reported that trying to distinguish between 'normal' adolescent behaviour and signs of mental health distress had proved very challenging. 
I don't know if accessing services in and of itself is problematic, it's more understanding what am I looking at? Do I need a medical professional's assistance here, or does this CHILD just need parenting? (daughter, 16 yrs)

\section{Stigma}

A small number of families reported their child had been resistant to treatment for fear of being ridiculed by peers. While no parents reported a reluctance to access help on account of stigma, this may be because the majority of families who presented to ED were already engaged with mental health services.

There were problems in that CHILD did not want to be seen as a girl that has mental health issues and needs to go to counselling which becomes obvious when she's taken out of class. (daughter, 14 yrs)

\section{An ideal service environment}

When asked what an ideal service environment might look like, the features discussed below were frequently echoed and were often solutions to the barriers parents had encountered.

\section{Systemic factors within healthcare \& schools}

Expanded services

Parents repeatedly appealed for more services, particularly parents of children and young people with chronic mental health issues and families living regionally. Some parents wanted more outreach services while others wanted more locally accessible inpatient treatment programs, particularly those from regional areas. Other parents wanted to see family therapy services made more widely available given the impact their child's illness was having on family dynamics. This was particularly so for parents who felt shut out of the child-clinician therapeutic relationship. 
... more services basically ... there is such a need for it, especially in regional areas ... they're so inundated that they can't help everybody. ...I tried to ring CAMHS again the other day and they pretty much said to me that unless he's got a plan and you know he's going to go ahead with it...don't call us back. (son, 14 yrs)

In the region I'm in, the missing service is that there is no adolescent therapeutic mental health <inpatient $>$ facilities. (son, 17 yrs)

When there's a child that's going through this sort of stuff, I think often the other siblings are forgotten. ... and I think it's really important that they get to express how it affects them and to know that they're still important and part of the family. (daughter, 15 yrs)

Improved access to services

Families consistently called more service availability both after hours and during school holiday periods.

I think when you've got kids with autism, things like that, you should be able to access a paediatrician pretty easily - like an everyday doctor. (son, 8 yrs)

Many parents appealed for a service to personally assist them in navigating available services.

There's lot of information out there, but a mental health nurse or someone like that who is dialed into the local area and knows what services are there and can get appointments for you pretty much straight away. A crisis isn't happening in two weeks' time, it's happening then. (daughter, 10 yrs)

Other parents called for improved communication between health providers in order to ensure their child was receiving optimal and efficient care.

I think the psychologists and paediatricians and <mental health services $>$ should be working more hand-in-hand; relaying the information around so that people call you and say, "Hey, I'm from X. I can do this for you .... What would you like?" But no. You ring up <a mental 
health service> and they say, "You need a referral for this or you need to go through a process." You don't have time for a process when it comes to crises like that. (son, 8 yrs)

A large majority of parents also felt strongly that basic mental health care should be made publicly available for all children andyoung people, without service limitations. For many families, capping of the Medicare-subsidised mental health care rebates to ten sessions per year severely impacted their child's access to ongoing care.

Respite and support

Many parents called for a substantial increase in child and adolescent respite support services. For the very few families who had benefited from this kind of support, parents had relished it while the children flourished. Parents were also keen to see more community-based peer support groups made available for children and adults, perhaps targeted for specific ages and for those with comorbidities.

I'm a single mum with a Dad that won't have my son overnight and he's limited support. It's exhausting. Absolutely exhausting. ... there's no opportunity for respite. (son, 17 yrs)

Supportive schools

From a parent perspective, a core component included schools supporting their child's mental health. Many parents envisaged public and private schools having an onsite, bulk billing psychologist accessible during school hours and teachers who had sufficient mental health knowledge to flag students they felt were struggling.

When we first started this journey, it was the school that really helped us. ... the school had a psychologist coming in once a fortnight ... they were communicating with me, opening my eyes to what I wasn't necessarily seeing in my busy world. (daughter, 14 yrs)

For others, their ideal system extended to schools adopting routine screening measures for mental health issues.

I think there should be a bit of a trigger point with the school where, if a student has missed $X$ amount of school, the Wellbeing process should kick in and that they're quite proactive about it. (daughter, 17 yrs) 


\section{Family/child factors}

Improved mental health education for parents

Many parents conceded that there was not enough parent-focused education on child mental health issues. Some called for a federally-driven mental health awareness initiative not only to reduce stigma but also to help parents distinguish between normal behaviour changes and symptoms of a mental health problem.

I find it difficult to get good information to know the difference between developmentally pretty typical or something a little outside of that that it's worth pursuing with a medical professional. (daughter, 16 yrs)

\section{DISCUSSION}

This is the first Australian study to document the perspective of parents, whose children have attended an ED for anxiety or depression, on the mental health care system, including barriers and ways to improve care. Presentations by children and young people to ED for mood and stress-related disorders, particularly depression and anxiety, have risen rapidly highlighting a need to understand whether community based-services are adequate. ${ }^{4}$ Parents reported multiple barriers including an overall lack of publicly funded community-based and inpatient services (specifically for children aged under 12 years), long service wait times and high outof-pocket costs, lack of school holiday and after-hours services, lack of information on how best to support their adolescent, and an unnavigable service system. Potential solutions included not only more services but specifically more Medicare funding for family therapy, more out-of-hours services, respite and peer support for more complex mental health problems, and a school based service offering onsite, bulk billing psychologists, and teachers trained to identify ‘at risk' students.

Our results are similar to those reported in Reardon's ${ }^{5}$ systematic review where common barriers identified included costs, long wait times and difficulty navigating the system. It extends these findings by reporting parent-identified solutions including sufficient service 
provision and flexible services in local community settings such as schools, increasing services during school holidays and out of hours, Medicare-funded family therapy sessions, and clinician provision of strategies for parents to best support their child's mental health.

Our study has several strengths. In addition to being the first of its kind, we purposively sampled across a variety of ED settings suggesting our findings may generalise to the wider population of families attending Victorian ED settings for child and adolescent anxiety or depression.

Use of phone and not face-to-face interviews, may have allowed for more open responses. . The study team developed a rigorous protocol and kept an audit trail of any changes. Regular meetings between the study team allowed for discussion and reflection on emerging themes.

Whilst we attempted to exclude children who had self-harmed (representing a more severe mental health presentation which may have affected a parent's experience of the mental health system), some parents did report that their child had suicidal ideation or self-harm around the time of their ED presentation. Two interviewers had a clinical background which potentially may have influenced the findings however, this was anticipated and addressed by rigorous training, weekly discussions and supervision from an experienced clinician researcher $(\mathrm{HH})$. Questions 4 and 10 of our interview guide may have been suggestive to parents.

\section{Conclusion}

Parents of children attending the ED for the common conditions of anxiety and depression are generally dissatisfied with community and inpatient services for child mental health, finding them to be in scarce supply, difficult to access, and unavailable after hours or during school holidays. Families, policy makers and clinicians must now work together to develop and test sustainable solutions to improve access to care, including improving parent mental health literacy to better recognise early mental health symptoms and support their child's behaviour and emotional difficulties at home; providing accessible information on available services 
including operating hours, costs and eligibility criteria; providing more services in schools; ensuring clinicians work with parents to enable them to support their child or young person at home; and providing respite. Without these solutions, we will likely see increasing presentations for anxiety and depression to our emergency departments.

\section{Acknowledgements}

We would like to thank all the families who volunteered their time for the study.

This article is protected by copyright. All rights reserved. 


\section{References}

1. Council of Australian Governments. The Fifth National Mental Health and Suicide Prevention Plan, 2017. Available at: http://www.coaghealthcouncil.gov.au/Portals/0/Fifth \%20National\%20Mental\%20Health\%20and\%20Suicide\%20Prevention\%20Plan.pdf (accessed 25 June 2019).

2. Department of Health and Human Services. Victoria's 10-Year Mental Health Plan, 2015. Available at: https://www2.health.vic.gov.au/about/publications/policiesandguidelines/ victorias-10-year-mental-health-plan (accessed 30 April 2019).

3. Reavley NJ, Jorm AF. Associations between beliefs about the causes of mental disorders and stigmatising attitudes: Results of a national survey of the Australian public. Australian \& New Zealand Journal of Psychiatry 2014;48(8):764-771.

4. Hiscock H, Neely RJ, Lei S, et al. Paediatric mental and physical health presentations to emergency departments, Victoria, 2008-15. Medical Journal of Australia 2018;208(8):343348.

5. Reardon T, Harvey K, Baranowska M, et al. What do parents perceive are the barriers and facilitators to accessing psychological treatment for mental health problems in children and adolescents? A systematic review of qualitative and quantitative studies. European Child \& Adolescent Psychiatry 2017;26(6):623-647.

6. Sawyer MG, Rey JM, Arney FM, et al. Use of health and school-based services in Australia by young people with attention-deficit/hyperactivity disorder. Journal of the American Academy of Child \& Adolescent Psychiatry 2004;43(11):1355-1363.

7. Boulter E, Rickwood D. Parents' experience of seeking help for children with mental health problems. Advances in Mental Health 2013;11(2):131-142.

8. Kallio H, Pietilä AM, Johnson M, Kangasniemi M. Systematic methodological review: Developing a framework for a qualitative semi-structur ed interview guide. Journal of Advanced Nursing. 2016: 72(12):2954-65.

9Australian Bureau of Statistics. 1270.0.55.005 - Australian Statistical Geography Standard (ASGS): Volume 5 - Remoteness Structure, July 2016. Published 2018. Table 3: Correspondence 2017 Postcode to 2016 Remoteness Area. Available at: 
https://www.abs.gov.au/AUSSTATS/abs@.nsf/DetailsPage/1270.0.55.005July\%202016? OpenDocument (accessed 25 June 2019).

10. Carpenter C. Phenomenology and Rehabilitation Research. In: Liamputtong P (ed) Research Methods in Health: Foundations for Evidence-Based Practice. Melbourne: Oxford University Press; 2010; pp.123-140.

11. Norlyk A, Harder I. What Makes a Phenomenological Study Phenomenological? An Analysis of Peer-Reviewed Empirical Nursing Studies. Qualitative Health Research 2010;20(3):420-431.

12.Elo S, Kyngas H. The qualitative content analysis process. Journal of Advanced Nursing 2008; 62(1): 107-115

13. Morse J, Richards L. Coding. In: Morse J and Richards L (eds) Read Me First for a User's Guide to Qualitative Methods. Thousand Oaks, CA: Sage; 2002; pp.111-128.

This article is protected by copyright. All rights reserved. 


\section{Tables}

\section{Table 1. Exclusion Criteria}

\section{Full exclusion critera}

1. Child did not present to ED with a parent or guardian;

2. Child presented following an episode of self-harm and/or had made a suicide attempt, thereby requiring medical treatment;

3. Parent had insufficient English;

4. Parent/carer had previously been contacted about the study; or

5. Postal address or phone number was not listed in the child's records.

6. Children in out-of- home care

This article is protected by copyright. All rights reserved. 


\section{Table 2. Interview Guide}

\section{Qualitative Interview Guide}

1. What led you to take <child's name $>$ to Emergency on this occasion?

2. What were you hoping the emergency department would be able to do for <child name $>$ ?

3. Before your recent visit, had you tried to access any other services for < child name's $><$ anxiety/depression $>$ ?

4. What do you think are some of the barriers/difficulties that prevented you from accessing/using other services?

5. Do you know about services aside from the hospital emergency that are available for children's mental health?

6. What sorts of things would help you feel really supported in the community to manage < child name's $>$ <anxiety/depression $>$ ? Are there changes you would make to existing services? Are there services missing that you would like to see made available?

7. What resources would be helpful for you?

8. What would a helpful service look like?

9. Having gone through your experience, what information do you wish you knew at the start? What tips would you pass on to someone/another family in your situation?

10. We know it can be hard to spot mental health problems in kids and we want to help parents become more aware of the signs and symptoms. How do you think we could best do this?

11. Where would you want to get this information from?

12. Is there anything <else> within family life that may have impacted your ability to get the help you needed for <child's name>? 


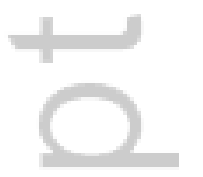

This article is protected by copyright. All rights reserved. 
Parent perspectives on children and young people's mental health services in Victoria what's wrong and how to fix it: A multi-site qualitative study.

Article Type: Original Article

\author{
Authors \\ O’Loughlin $^{1,2}$, Stephanie J Brown ${ }^{1,3}$, David M Krieser ${ }^{1,3,4}$, Adam West ${ }^{5}$, Pauline Chapman ${ }^{6}$, \\ Robyn Lawford ${ }^{1,2}$, John A Cheek ${ }^{1,2,3}$

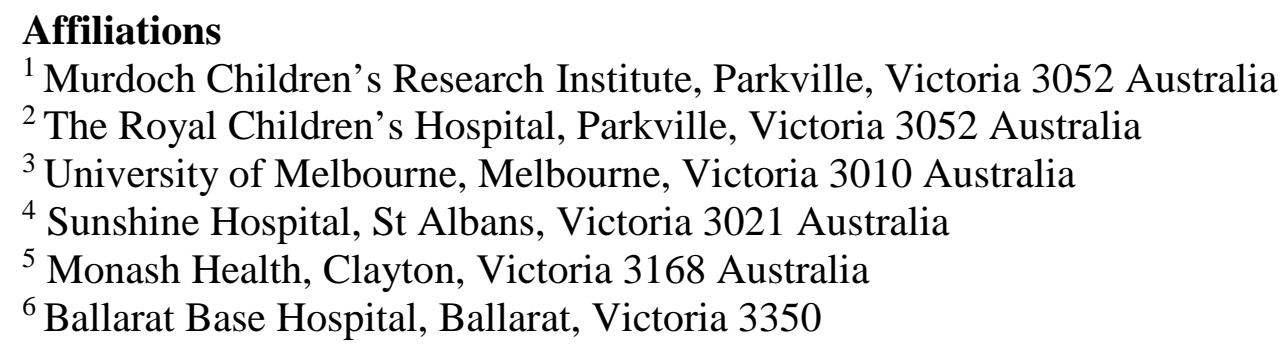

Harriet Hiscock ${ }^{1,2,3}$, Ann-Siobhan Connolly ${ }^{1}$, Katie Dunlop ${ }^{1}$, Prescilla Perera ${ }^{1,2}$, Rachel

Correspondence to: Prof Harriet Hiscock

Director, Health Services Research Unit

The Royal Children's Hospital

50 Flemington Road

Parkville, Victoria, 3052, Australia

(E): harriet.hiscock@rch.org.au

(T): $0393456910(\mathrm{M}): 0438420116$

\title{
Declaration of Conflicting Interests
}

The authors declare no potential conflicts of interest with respect to the research, authorship and/or publication of this article.

\section{Funding support}

The study is funded by a Royal Children's Hospital Foundation grant (2017-892). Professor Hiscock is supported by a National Health and Medical Research Council (NHMRC) Practitioner Fellowship (1136222) and Professor Brown is supported by a NHMRC Senior Research Fellowship (1103976). The MCRI is supported by the Victorian Government's Operational Infrastructure Support Program. Funders had no role in data collection, interpretation and reporting of findings of the study.

\section{Author contributions}

Conceptualization and design: H.H, R.O, S.J.B, D.M.K, A.W, P.C and J.A.C.; acquisition, analysis or interpretation of data: A.S.C, K.D, P.P and R.L; Writing -original draft preparation: A.S.C and P.P; Writing- review and editing: A.S.C, P.P, H.H, S.J.B, R.O, D.M.K, K.D, P.C, A.W, R.L, J.A.C 


\section{University Library}

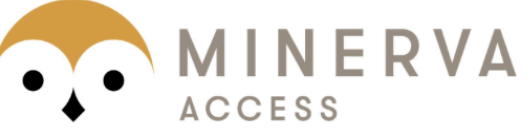

A gateway to Melbourne's research publications

Minerva Access is the Institutional Repository of The University of Melbourne

\section{Author/s:}

Hiscock, H;Connolly, A-S;Dunlop, K;Perera, P;O'Loughlin, R;Brown, SJ;Krieser, DM;West, A;Chapman, P;Lawford, R;Cheek, JA

Title:

Parent perspectives on children and young people's mental health services in Victoria What's wrong and how to fix it: A multi-site qualitative study

Date:

2020-02-26

\section{Citation:}

Hiscock, H., Connolly, A. -S., Dunlop, K., Perera, P., O'Loughlin, R., Brown, S. J., Krieser, D. M., West, A., Chapman, P., Lawford, R. \& Cheek, J. A. (2020). Parent perspectives on children and young people's mental health services in Victoria - What's wrong and how to fix it: A multi-site qualitative study. JOURNAL OF PAEDIATRICS AND CHILD HEALTH, 56 (7), pp.1114-1120. https://doi.org/10.1111/jpc.14835.

Persistent Link:

http://hdl.handle.net/11343/275462 